\title{
Low frequency admittance of quantized Hall conductors
}

\author{
T. Christen and M. Büttiker \\ Université de Genève, 24 Quai E. Ansermet \\ CH-1211 Genève, Suisse
}

\begin{abstract}
We present a current and charge conserving theory for the low frequency admittance of a two-dimensional electron gas connected to ideal metallic contacts and subject to a quantizing magnetic field. In the framework of the edge-channel picture, we calculate the admittance up to first order with respect to frequency. The transport coefficients in first order with respect to frequency, which are called emittances, determine the charge emitted into a contact of the sample or a gate in response to an oscillating voltage applied to a contact of the sample or a nearby gate. The emittances depend on the potential distribution inside the sample which is established in response to the oscillation of the potential at a contact. We show that the emittances can be related to the elements of an electro-chemical capacitance matrix which describes a (fictitious) geometry in which each edge channel is coupled to its own reservoir. The particular relation of the emittance matrix to this electro-chemical capacitance matrix depends strongly on the topology of the edge channels: We show that edge channels which connect different reservoirs contribute with a negative capacitance to the emittance. For example, while the emittance of a two-terminal Corbino disc is a capacitance, the emittance of a two-terminal quantum Hall bar is a negative capacitance. The geometry of the edge-channel arrangement in a many-terminal setup is reflected by symmetry properties of the emittance matrix. We investigate the effect of
\end{abstract}


voltage probes and calculate the longitudinal and the Hall resistances of an ideal four-terminal Hall bar for low frequencies. 


\section{INTRODUCTION}

The quantized Hall effect [1] provides particularly interesting tests of our understanding of electrical transport. Application of a resistance formula which treats all contacts to a two-dimensional electron gas on equal footing [2] has considerably revised the traditional picture of the quantized Hall effect and has led to the successful explanation of many novel experiments [3]. It is the purpose of this work to approach the low frequency electrical transport in two-dimensional electron systems (2DES) subject to strong magnetic fields from a similar point of view. In contrast to the dc-transport properties, which have become increasingly well understood, the ac-transport properties have found much less attention. However, a charge and current conserving theory for the low frequency admittance $G_{\alpha \beta}(\omega)$ of a general arrangement of mesoscopic conductors has recently been worked out [4,5]. We apply this theory to Hall systems in the integer quantum Hall regime at a plateau. A charge and current conserving theory requires knowledge of the non-equilibrium potential distribution inside the conductor. In the quantum Hall regime the determination of this potential becomes simple due to the formation of edge channels [6]. As discussed in detail by Chklovskii et al. [7] and closely related works [8 14], there occurs a decomposition of a 2DES in metal-like edge channels and dielectric-like regions. Consequently, the non-equilibrium potential is also determined by the properties of the edge channels. If the edge channels behave like perfect metals they screen any excess charge. The resulting non-equilibrium potential is determined by the geometry of the edge-channel arrangement alone. On the other hand, if the charge in the edge channels is not perfectly screened the non-equilibrium potential depends on the density of states of the edge channels. The resulting potential distribution is not of geometrical nature alone but contains quantum corrections due to the finite density of states of the edge channels. It is of particular interest to investigate to what extent such quantum corrections affect the dynamic transport properties of a 2DES.

The admittance $G_{\alpha \beta}(\omega)$ gives the linear current response $\delta I_{\alpha} \exp (-i \omega t)$ at a contact $\alpha$ of a device, if at contact $\beta$ a voltage oscillation $\delta V_{\beta} \exp (-i \omega t)$ is applied: 


$$
\delta I_{\alpha}(\omega)=\sum_{\beta} G_{\alpha \beta}(\omega) \delta V_{\beta}(\omega)
$$

The voltage variation $\delta V_{\beta}$ is related to the variation of the electro-chemical potential $\delta \mu_{\beta}$ in reservoir $\beta$ by $\delta \mu_{\beta}=e \delta V_{\beta}$, where $e$ is the electron charge. The theory [4, $[$ ] deals with the dcconductance, $G_{\alpha \beta}^{(0)}$, and the first-order term with respect to frequency, $E_{\alpha \beta} \equiv i\left(d G_{\alpha \beta} / d \omega\right)_{\omega=0}$, which is called the emittance matrix. The low frequency admittance can then approximately be written in the form

$$
G_{\alpha \beta}(\omega)=G_{\alpha \beta}^{(0)}-i \omega E_{\alpha \beta}
$$

For an array of macroscopic conductors of which each is connected to a single contact, the emittance is just a geometrical capacitance, i.e. $E_{\alpha \beta}=C_{\alpha \beta}$. However, this is not true for mesoscopic conductors and conductors which connect different reservoirs [5]. Firstly, it is not the geometrical capacitance but rather the electro-chemical capacitance which relates charges at mesoscopic conductors with voltage variations in the reservoirs. Secondly, conductors which connect different reservoirs allow a transmission of charge which leads to inductance-like contributions to the emittance.

We shall show that the emittance $E_{\alpha \beta}$ of a quantized Hall sample is the sum over elements of the electro-chemical capacitance matrix, $c_{\mu, k l}$, for edge-channels $l$ into which charge is injected at contact $\beta$ and for edge-channels $k$ from which charge is emitted into contact $\alpha$. The electro-chemical capacitance matrix, $c_{\mu, k l}$, is determined by considering each edge channel as a metal strip connected to a single contact. Our expression for the emittance is simple enough in order to discuss arbitrarily complicated edge-channel arrangements without much technical effort, once the electro-chemical capacitance matrix of the edge-channel arrangement is known. We emphasize here that our theory satisfies charge and current conservation which are due to a perfect screening of electric fields in the reservoirs and in the gates used to form the conductor. Current conservation implies that the admittance satisfies $\sum_{\beta} G_{\alpha \beta}=\sum_{\alpha} G_{\alpha \beta}=0$.

Two simple geometries can be used to illustrate the different behavior of the emittance, namely the Hall bar geometry (Fig. 1.a), and the Corbino geometry (Fig. 1.b). We will show 
that in a Hall bar kinetic charge motion of electrons along the edge channels dominates the Coulomb interaction between the reservoirs. The emittance is a negative electro-chemical capacitance, i.e. $E=-C_{\mu}$, with $C_{\mu}>0$. On the other hand, in the Corbino geometry contacts are located at the inner and the outer perimeter of an annular film [15,16]. Hence, edge channels do not connect different reservoirs and will thus not contribute to a dc-current. Moreover, in contrast to the bar geometry in the Corbino disc capacitive effects dominate and the emittance is a capacitance, i.e. $E=C_{\mu}$.

The transverse potential profile in a cross section of these conductors is qualitatively shown shown in Fig. 2 which is to be discussed below. Here we only mention that the similarity of this potential for the two different setups applies only to the bulk of the sample. We will assume in this work that the capacitances and emittances are dominated by the bulk and that contact capacitances can be neglected.

The paper is organized as follows. In Sect. 巴I we briefly recall the edge-channel picture of a 2DES at equilibrium. In Sect. III, we discuss the dc-nonequilibrium electric potential in terms of an electro-chemical capacitance matrix, and the expression for the dc-conductance $G_{\alpha \beta}^{(0)}$ is derived. In Sect. $\mathbb{\nabla}$ we outline the theory of emittances and derive an expression for the emittance matrix $E_{\alpha \beta}$ for quantized Hall samples. The result is applied to various specific examples in Sect. V. In Sect. V1 we investigate the effect of a voltage probe. As an

application, we calculate in Sect. VII the longitudinal resistance and the Hall resistance of a four-probe quantum Hall bar for low frequencies.

\section{QUANTIZED HALL SAMPLES AT EQUILIBRIUM}

We begin with a brief discussion of important equilibrium properties of a 2DES at an (integer) Hall plateau. Consider the two-terminal quantum Hall bar in Fig. 1.a. The bar is connected on either side via ideal contacts to particle reservoirs $\alpha=1,2$ at electro-chemical potentials $\mu_{\alpha}=E_{F, \alpha}+e U_{\alpha}$. Here, $E_{F, \alpha}$ and $U_{\alpha}$ denote the chemical and the electric potential 
of reservoir $\alpha$, respectively. The strong magnetic field is assumed to be perpendicular to the plane of the 2DES. Translational invariance of the potential $e U(x)$ in the $y$-direction allows one to restrict the considerations to a transverse cross section of the sample. The single-particle potential as a function of $x$ is sketched in Fig. 2. a for the equilibrium case where the reservoirs are kept at equal electro-chemical potential, say $\mu=E_{F}$ where $U_{\alpha} \equiv 0$. For the moment, we assume that the Fermi level lies in the region between the extended bulk states of the first and the second (spin-split) Landau levels. Hence, in the bulk the states of a single Landau level are completely filled (black dots in Fig. 2.a). At the sample boundary, however, the confinement potential strongly bends up the single-particle potential which, therefore, intersects the Fermi energy. This leads to the existence of extended states at the Fermi level (edge channels) along the sample boundary. For non-interacting electrons [6] the intersection of the single particle energy with the Fermi energy is sharp. The transverse size of an edge channel is of the order of a magnetic length, $l_{m}=\sqrt{\hbar /|e B|}$. The mean drift velocity of a carrier with coordinate $x$ points in $y$-direction and is given by [17] $v(x)=(d U / d x) / B$. This is just the Lorentz drift of the center of a cyclotron orbit in an electric field.

In a quantized Hall sample a current density exists which is a pure equilibrium phenomenon and cannot lead to a current between reservoirs. For a filled Landau level, the diamagnetic current density can be written in the form 17

$$
j=-\frac{e^{2}}{h} \frac{d U}{d x}
$$

The total current through a contact is obtained by a transverse spatial integral of $j(x)$. It vanishes at equilibrium since at both boundaries of integration, $\mu=E_{F}+e U_{k}$ holds, where $k=1,2$ labels the edge channels. Of course, this statement is valid independent of the geometrical arrangement of the edge channels as long as the cross section is constructed such that all edge channels of a contact are included. In particular, it is independent of the specific space dependence of the equilibrium potential which can be very complicated.

The inclusion of Coulomb interaction, even within a mean-field approximation, dras- 
tically affects the results of the single-particle approach. Coulomb interactions lead to an electro-static restructuring of the edge $[7$ 12]. The 2DES is composed of alternating strips of compressible and incompressible electron liquids with finite widths. Incompressible regions where the filling factor has discrete values behave like dielectrics. Quantitative analytical predictions of the widths of the compressible and incompressible strips have been made by Chklovskii et al. [7]. These predictions are in good agreement with numerical work by Lier and Gerhardts [9]. Edge channels correspond to the compressible regions where singleparticle states are partially filled and the electric potential is pinned to the Fermi level (flat parts in Fig. 2.a). Edge channels have screening properties similar to metallic strips. The many-particle effects become important if the strength $d U / d x$ of the (unscreened) confinement field is weaker than the characteristic electric field of electron-electron interaction, i.e. if $\alpha \equiv|d U / d x| 4 \pi \epsilon_{0} \epsilon_{r} l_{m}^{2} / e \ll 1$, where $\epsilon_{0} \epsilon_{r}$ is the dielectric constant. The strength of the confinement field depends on the specific fabrication of the boundaries of the 2DES under consideration (etching, gates, etc.). It has been argued that compressible and incompressible strips can even become comparable in size [7]. Interaction-dominated edge channels are useful in the theory of the fractional quantum Hall effect [11, 112, 18, 19]. However, the structure of fractional edge channels is much more complicated, and we shall restrict our considerations to the integer quantum Hall regime.

In the following considerations, three quantities which characterize the equilibrium state of a sample are important in order to discuss low frequency transport close to equilibrium. First, the density of states, $d N_{k} / d E$, of the edge channel $k$ at the Fermi level gives the change in the number of states if the electro-chemical potential is varied for fixed electrostatic potential (i.e. fixed band bottom). For non-interacting electrons this density of states is determined by the (equilibrium) velocity of carriers $v(s)=\left(d U_{e q}(s) / d x\right) / B$ along the the path $s$ of the edge channel, where $x$ is now the transverse coordinate. It is given by $d N_{k} / d E=\int d s / h v$, where the integral over $s$ is along the entire path of the edge channel from one sample contact to the other. For the interacting model this density of states diverges at 
$k T=0$ since the single particle potential is flat. But the density of states is finite for any non-vanishing temperature. A finite DOS of edge channels due to a considerable suppression of screening at small temperatures is indicated by the numerical results presented by Lier and Gerhardts [9].

Secondly, one can attribute to the arrangement of metal-like edge channels a geometrical capacitance matrix $c_{j k}$. For given geometry, this matrix can in principle be derived with the help of Poisson's equation. For metallic screening this capacitance matrix is determined by the width and location of the edge channels. It is, therefore, also a function of the magnetic field and the electro-chemical potentials applied to the contacts and the gates [20].

We finally take into account that each edge channel connects reservoirs in a directed way, due to the uni-directional velocity of the carriers. This connection is determined by the transmission and reflection probability of the contact. In the following, we shall always regard the just mentioned characteristics of the equilibrium state to be given.

\section{THE NONEQUILIBRIUM STEADY STATE}

\section{A. Electro-chemical capacitance of edge channels}

Consider the two-terminal bar of Fig. 1. 1.a under nonequilibrium conditions. A cross section of the single-particle potential in the nonequilibrium case is shown in Fig. 2.b. A small increase of the voltage $\delta V_{\beta}$ at $\operatorname{contact} \beta$, say $\beta=1$, implies an electro-chemical voltage shift $\delta V_{k}$ in channel $k$. If the transmission probability from the contact into the edge channel is 1 , then the chemical potential shift of that edge channel is the same as that of the reservoir $\delta V_{k}=\delta V_{\beta}$. As a consequence, into the edge channel a charge is injected which is proportional to the density of states (DOS) $d N_{k} / d E$ of the edge channel $k$. This added charge creates in the whole sample an electric nonequilibrium potential which shifts the band bottom. This

leads, in turn, to the injection of screening charge. The total charge $\delta q_{k}$ in edge channel $k$ is then given by 


$$
\delta q_{k}=D_{k}\left(\delta V_{k}-\delta U_{k}\right)
$$

where $D_{k}=e^{2} d N_{k} / d E$ is the quantum capacitance of edge channel $k$. The nonequilibrium electric potential $\delta U_{k}$ of edge channel $k$ can be calculated for a given charge distribution by solving the electrostatic boundary-value problem associated with Poisson's equation. This leads to the introduction of the geometrical capacitance matrix $c_{k j}$ of the edge-channel configuration by $\delta q_{k}=\sum_{j} c_{k j} \delta U_{j}$. Note, that the $c_{k j}$ are calculated for edge channels which are disconnected from the contacts and where charge is not conserved. But the relevant (gauge invariant) potentials are the electro-chemical potentials and not the electrostatic potentials of disconnected edge channels. We define thus an electro-chemical capacitance matrix $c_{\mu, k j}$ by $[4$

$$
\delta q_{k}=\sum_{j} c_{\mu, k j} \delta V_{j}
$$

considering charge conservation, $\sum \delta q_{i}=0$, which is due to the connection to the reservoirs. One finds then from Eqs. (41) and (5) $c_{\mu, 11}=c_{\mu, 22}=-c_{\mu, 12}=-c_{\mu, 21} \equiv c_{\mu}$, where the relative electro-chemical capacitance $c_{\mu}$ of the two edge channels is given by

$$
c_{\mu}^{-1}=c_{0}^{-1}+D_{1}^{-1}+D_{2}^{-1}
$$

This describes the relative geometrical capacitance [21], $c_{0}=\left(c_{11} c_{22}-c_{12}^{2}\right) /\left(c_{11}+2 c_{12}+c_{22}\right)$, in series with the quantum capacitances $D_{k}$.

As an example, we consider the non-interacting case where the widths $\xi_{k}$ of the edge channels $k=1,2$ of length $L_{y}$ are very small (i.e. $\xi_{k} \approx l_{m}$ ). For the sake of simplicity, we assume them to be equal to each other, $\xi_{k} \equiv \xi$. The distance between the edge channels is denoted by $L_{x}$, and the charge is to be uniformly distributed in the edge channels. For line charges, the geometrical capacitance becomes [22] $c_{0}=\left(L_{y} \pi \epsilon_{0} \epsilon_{r}\right) /\left(1+\ln \left(L_{x} / \xi\right)\right)$. The density of states $D_{k}$, on the other hand, are given by $D_{k}=L_{y} e /\left(2 \pi l_{m}^{2}\left|d U_{k} / d x\right|\right)$, where we assumed very steep confinement potentials $d U_{k} / d x=(d U / d x)_{x_{k}}$ at the edge channels located at $x_{k}$. The electro-chemical capacitance $c_{\mu}$ can then be written as 


$$
c_{\mu}=\frac{\pi \epsilon_{0} \epsilon_{r} L_{y}}{1+\ln \left(L_{x} / \xi\right)+\pi\left(\alpha_{1}+\alpha_{2}\right) / 2}
$$

Here $\alpha_{k}=\left|d U_{k} / d x\right| 4 \pi \epsilon_{0} \epsilon_{r} l_{m}^{2} / e$ is the ratio between the confinement field and the interaction field at the edge channel $k$. To be consistent with the non-interacting case, one must have $\alpha_{k} \gg 1$. Note that $c_{\mu}$ depends on the magnetic field via $\alpha_{k} \propto 1 / B$, and via the $B$-dependence of the geometry of the edge-channel arrangement.

Charge conservation in the sample is reflected by the sum rule

$$
\sum_{k} c_{\mu, k l}=\sum_{l} c_{\mu, k l}=0
$$

which is a well-known property of a set of capacitors where ground is included. One concludes that two-terminal systems are particularly simple since $2 \times 2$-matrices satisfying Eq. (8) are characterized by a single quantity and have thus purely scalar properties. We will see later on that equations analogous to Eq. (8) hold also for the dc-conductance and the emittance [5.23]. Below it will be important that the electro-chemical capacitance matrix is symmetric and an even function of the magnetic field, i.e. $c_{\mu, k l}(B)=c_{\mu, l k}(B)$ and $c_{\mu, k l}(B)=c_{\mu, k l}(-B)$, respectively. These properties are evident from our definition of $c_{\mu, k l}$.

\section{B. DC-conductance for a two-terminal Hall bar}

To find the dc-conductance in the transmission approach the current can be evaluated in response to a small variation of the chemical potential of the contacts keeping the electrostatic potential fixed at its equilibrium value. The transmission probabilities are a functional of the equilibrium electrostatic potential only. Here we briefly discuss the derivation of the dc-conductance using the actual non-equilibrium current. For a detailed discussion of the various possible definitions of currents and their physical interpretation we refer the reader to Komiyama and Hirai [24]. In order to find the dc-conductance $G_{\alpha \beta}^{(0)}$ of the bar in Fig. 11.a, we remark that the total nonequilibrium current through a contact consists of two contributions 25],26. At contact 1, for example, a first part $\delta I_{1}^{(u)} \equiv\left(e^{2} / h\right)\left(\delta U_{1}-\delta U_{2}\right)$ originates from the 
action of the nonequilibrium electric field on the occupied equilibrium states in the Landau level. This part is obtained from a spatial integration of the current density (3) in the region between the edge channels. A second part $\delta I_{1}^{(q)} \equiv\left(\delta q_{1} v_{1}+\delta q_{2} v_{2}\right) / L_{y}$ is caused by the motion of the added charge density $\delta q_{k} / L_{y}$ with an equilibrium velocity $v_{k}$ in edge channel $k$. In the present notation, the relation between the velocity and the DOS for quasi one-dimensional conductors reads $v_{k}= \pm L_{y} e^{2} /\left(h D_{k}\right)$ where the sign is different for opposite edge channels. Using this and Eq. (田) gives $\delta I_{1}^{(q)}=\left(e^{2} / h\right)\left(\delta V_{1}-\delta U_{1}\right)-\left(e^{2} / h\right)\left(\delta V_{2}-\delta U_{2}\right)$. It follows immediately that the total current $\delta I_{1}=\delta I_{1}^{(u)}+\delta I_{1}^{(q)}$ depends only on the electro-chemical potentials of the contacts and is given by $\delta I_{1}=\left(e^{2} / h\right)\left(\delta V_{1}-\delta V_{2}\right)$ with a zero-frequency conductance $G^{(0)} \equiv G_{11}^{(0)}=G_{22}^{(0)}=-G_{12}^{(0)}=-G_{21}^{(0)}=e^{2} / h$. This universal result reflects the integer quantum Hall effect [1]. Using $\delta q_{1}=c_{0}\left(\delta U_{1}-\delta U_{2}\right)$ and $\delta q_{1}=c_{\mu}\left(\delta V_{1}-\delta V_{2}\right)$ with $c_{\mu}$ given by Eq. (6) we find for the ratio of the two currents $\delta I^{(q)} / \delta I^{(u)}=\left(D_{1}^{-1}+D_{2}^{-1}\right) c_{0}$. This ratio is large for small DOS $D_{k}$ of the edge channels, i.e. for a sufficiently steep slope of the confinement potential, when the chemical contribution to the current predominates. On the other hand, if the edge channels are macroscopic metallic conductors with complete screening, $\left(\delta U_{\alpha} \rightarrow \delta V_{\alpha}\right.$ and $\left.v_{k} \rightarrow 0\right)$ the chemical contribution vanishes and the electrostatic contribution predominates.

\section{DC-conductance for an $M$-terminal sample with $N$ edge channels}

Consider now a more general quantum Hall sample with $M$ contacts and $N$ edge channels. We assume that the density of states, $D_{k}$, and the electro-chemical capacitance matrix, $c_{\mu, k j}$ $(k, j=1, \ldots, N)$, are known. An expression for $c_{\mu, k j}$ in terms of the geometrical capacitance, $c_{k j}$, and the DOS of the edge channels, $D_{k}$, is derived in an appendix. Eqs. (4) and (5) are still valid in the present case. Each edge channel $k$ is connected to reservoirs $\alpha$ and $\beta$, where $\alpha=\beta$ is permitted. Reservoir $\beta$ injects carriers into edge channel $k$ from which carriers are emitted into reservoir $\alpha$. For simplicity and to be definite, we assume that the contact resistances of this sample are quantized [27,28. The transmission probability of a 
carrier in contact $\beta$ to enter edge channel $k$ is denoted by $\Delta_{k \beta}(B)$ and for quantized contact resistances is given by

$$
\Delta_{k \beta}(B)=\left\{\begin{array}{cc}
1 & \text { if contact } \beta \text { injects into channel } k \\
0 & \text { otherwise }
\end{array} .\right.
$$

Similarly, we introduce the probability of a carrier which approaches contact $\beta$ on an edge channel $k$ to enter the contact

$$
\Delta_{\alpha l}(B)=\left\{\begin{array}{cc}
1 & \text { if channel } l \text { emits into contact } \alpha \\
0 & \text { otherwise }
\end{array} .\right.
$$

From the micro-reversibility properties of the transmission probabilities we have $\Delta_{\beta k}(B)=$ $\Delta_{k \beta}(-B)$. The transmission probability of the contact plays the role of a topological factor determined by the connectivity of the edge channel to the contacts of the sample. With the help of the contact transmission probability (9), the variation $\delta V_{k}$ of the electro-chemical voltage of edge channel $k$ can be expressed in terms of the voltages $\delta V_{\beta}$ in the reservoirs:

$$
\delta V_{k}=\sum_{\beta=1}^{M} \Delta_{k \beta} \delta V_{\beta}
$$

The charge $\delta q_{k}$ in edge channel $k$ is then related to voltage variations in the contacts by

$$
\delta q_{k}=\sum_{\beta=1}^{M} \sum_{l=1}^{N} c_{\mu, k l} \Delta_{l \beta} \delta V_{\beta}
$$

The total charge in all those channels into which contact $\alpha$ injects is $\sum_{k} \delta q_{k} \Delta_{k \alpha}$. If there is no transmission (i.e. if all edge channels are connected to a single contact), one has $\Delta_{k \alpha}=\Delta_{\alpha k}$. A capacitance measurement yields then a capacitance matrix

$$
C_{\mu, \alpha \beta}=\sum_{k, l=1}^{N} \Delta_{k \alpha} c_{\mu, k l} \Delta_{l \beta}
$$

Below, we shall see that the assumption of the absence of transmission between different contacts is crucial in order to find a magneto-capacitance according to Eq. (13).

The dc-conductance $\delta I_{\alpha} / \delta V_{\beta}$ can be calculated following the same lines as above for the two-terminal case with a single Landau level. The current $\delta I_{\alpha}$ through contact $\alpha$ is obtained from a sum over all incoming and outgoing channels $k$ with a contribution $\delta I_{k}^{(q)}$ and over all Landau levels with a contribution $\delta I_{k}^{(u)}$. The well-known result [2] reads in our notation 


$$
G_{\alpha \beta}^{(0)}=\frac{e^{2}}{h}\left(K_{\beta} \delta_{\alpha \beta}-\sum_{k} \Delta_{\alpha k} \Delta_{k \beta}\right) .
$$

For the derivation of Eq. (14) we used that $\sum_{k} \Delta_{k \beta} \Delta_{k \alpha}=K_{\beta} \delta_{\alpha \beta}$ where $K_{\beta}$ is the number

of edge channels in which contact $\beta$ injects. The diagonal element, $G_{\beta \beta}^{(0)}$, is $e^{2} / h$ times the number of channels which leave contact $\beta$ and which do not return to this contact, and $-G_{\alpha \beta}^{(0)}$ $(\alpha \neq \beta)$ is $e^{2} / h$ times the number of directed channels going from contact $\beta$ to contact $\alpha$. Note that both the current conservation property $\sum_{\alpha} G_{\alpha \beta}^{(0)}=\sum_{\beta} G_{\alpha \beta}^{(0)}=0$ and the OnsagerCasimir reciprocity relations, $G_{\alpha \beta}^{(0)}(-B)=G_{\beta \alpha}^{(0)}(B)$, are satisfied.

\section{THE EMITTANCE MATRIX}

\section{A. Emittance matrix for general mesoscopic conductors}

First, we recall the theory of the emittance for a general arrangement of mesoscopic conductors by closely following Ref. [5]. Once the electrostatic geometrical capacitance matrix is known, we can formulate our discussion in terms of a discrete set of potentials which we take to be constants along each edge channel. A general formulation of the theory for such a discrete potential model is the subject of Ref. [29]. It is well-known that the transmission approach to current transport relates conductances to scattering matrices of the conductors. A scattering matrix relates incoming and outgoing current amplitudes of the contacts $\alpha=1, \ldots, M$ of a sample for each conduction channel $k=1, \ldots, N$. The DOS $d N_{k} / d E$ of channel $k$ expressed in terms of the scattering matrix can then be written as a sum of partial densities of states, $d N_{\alpha k \beta} / d E$. The quantity $d N_{\alpha k \beta} / d E$ is the DOS of channel $k$ associated with carriers which are scattered from contact $\beta$ to contact $\alpha$. A slight variation $\delta V_{\beta}$ of the voltage in contact $\beta$ causes the injection of a total charge $\delta Q_{\alpha}$ through contact $\alpha$. Thus, a slowly oscillating voltage implies an additional current $-i \omega \delta Q_{\alpha} \exp (-i \omega t)$ at this contact. Now, it follows from the definition in Eq. (目) that the emittance $E_{\alpha \beta}$ can be 
identified with $\delta Q_{\alpha} / \delta V_{\beta}$. Note that there are two contributions to $\delta Q_{\alpha}$. A first part which neglects screening is given by a kinetic contribution

$$
\delta Q_{\alpha}^{(k)}=e^{2} \sum_{k=1}^{N} \sum_{\beta=1}^{M} \frac{d N_{\alpha k \beta}}{d E} \delta V_{\beta} .
$$

This part gives the charge which is scattered from the contacts $\beta$ to contact $\alpha$ due to the shift of the electro-chemical potentials $\delta V_{\beta}$ at fixed electrostatic potentials $\delta U_{k}$. However, the nonequilibrium electrostatic potential which is due to the nonequilibrium charge-distribution in the edge channels is still neglected in Eq. (15). In order to take it into account, we recall that the $\delta U_{k}$ are shifts of the band bottoms of the edge channels, which cause an induction of additional screening charges. Hence, there is a second contribution $\delta Q_{\alpha}^{(s)}$ given by the part of the screening charge which is eventually scattered to contact $\alpha$. It can be expressed in the form

$$
\delta Q_{\alpha}^{(s)}=-e^{2} \sum_{k=1}^{N}\left(\sum_{\beta=1}^{M} \frac{d N_{\alpha k \beta}}{d E}\right) \delta U_{k}
$$

The quantity in the large bracket, $d N_{\alpha k} / d E \equiv \sum_{\beta=1}^{M} d N_{\alpha k \beta} / d E$, is a partial DOS associated with carriers in channel $k$ emitted into contact $\alpha$ irrespective of the injecting contact $\beta$. The change of the electric potential $\delta U_{k}$ at channel $k$ is determined by the variations of the electro-chemical potentials of the conductors. Within linear response theory we write

$$
\delta U_{k}=\sum_{\beta=1}^{M} u_{k \beta} \delta V_{\beta}
$$

where the characteristic potentials $u_{k \beta}$ [5] give the change of the electrostatic potential of conductor $k$ if the voltage is changed in contact $\beta$ by unity. The sum of the two parts given by Eqs. (15) and (16) leads finally to the emittance matrix $\delta Q_{\alpha} / \delta V_{\beta}$ :

$$
E_{\alpha \beta}=e^{2} \sum_{k=1}^{N}\left(\frac{d N_{\alpha k \beta}}{d E}-\frac{d N_{\alpha k}}{d E} u_{k \beta}\right)
$$

The occurrence of the characteristic potentials $u_{k \beta}$ indicates the necessity of the knowledge of the nonequilibrium state in order get the emittance. Since the characteristic potentials are sample specific, one cannot expect to obtain a universal result for the ac-admittance. 
Furthermore, since the kinetic part and the screening part contribute with opposite signs, the emittance elements can have positive or negative sign depending on which part is dominant [5].

\section{B. Emittance matrix for quantized Hall samples}

To apply the result (18) to quantized Hall samples, one uses the fact that the partial DOS can be expressed in terms of the transmission probabilities $\Delta_{\alpha l}, \Delta_{k \beta}$, and the density of states $D_{k}$ of edge channel $k$ :

$$
e^{2} \frac{d N_{\alpha k \beta}}{d E}=\Delta_{\alpha k} D_{k} \Delta_{k \beta} \quad, \quad e^{2} \frac{d N_{\alpha k}}{d E}=\Delta_{\alpha k} D_{k}
$$

This follows directly from the suppression of backscattering in an edge channel. The characteristic potentials $u_{k \beta}$ follow from Eqs. (4), (5) and (11):

$$
u_{k \beta}=\sum_{l=1}^{N}\left(\delta_{k l}-D_{k}^{-1} c_{\mu, k l}\right) \Delta_{l \beta} .
$$

By inserting Eqs. (19) and (20) in Eq. (18) one obtains

$$
E_{\alpha \beta}=\sum_{k, l=1}^{N} \Delta_{\alpha k} c_{\mu, k l} \Delta_{l \beta} .
$$

This is the key result of our work. The emittance is the sum of all those charges which are emitted at contact $\alpha$ due to the injection of charge at contact $\beta$ mediated by Coulomb interaction between edge channels. The elementary process which contributes to the emittance is illustrated by the diagram in Fig. 3 .

The emittance has the following properties. From $\sum_{\alpha} \Delta_{k \alpha}=1$ and Eq. (8) one concludes that

$$
\sum_{\beta} E_{\alpha \beta}=\sum_{\alpha} E_{\alpha \beta} \equiv 0
$$

which is a consequence of charge neutrality [5]. Since $\Delta_{\alpha k}(-B)=\Delta_{k \alpha}(B)$, the OnsagerCasimir reciprocity relations [5] 


$$
E_{\alpha \beta}(B)=E_{\beta \alpha}(-B),
$$

based on micro-reversibility are satisfied, too. In contrast to $c_{\mu, k l}(B)$, the emittance matrix $E_{\alpha \beta}(B)$ is in general not symmetric. A comparison of the Eqs. (13) and (21) implies that the emittance is a (symmetric) capacitance, i.e. $E_{\alpha \beta} \equiv C_{\mu, \alpha \beta}$, if each edge channel $k$ is connected to a single reservoir, i.e. if $\Delta_{k \alpha}(B) \equiv \Delta_{\alpha k}(B)$ holds.

\section{EXAMPLES}

In this section we apply the previous results to various examples of Hall devices. The electro-chemical capacitance matrix $c_{\mu, k j}$ is always assumed to be known.

\section{A. Two-terminal devices}

The two-terminal devices in Figs. 1 and 1 . a can be characterized by the scalar admittance $G=G^{(0)}-i \omega E \equiv G_{11}=G_{22}=-G_{12}=-G_{21}$. While $G^{(0)}=e^{2} / h$ for the Hall bar in Fig.

1. $\mathrm{a}$, the dc-conductance vanishes identically for the Corbino disc in Fig. [1.b since there is no dc-current flowing through the contacts. From Eq. (21) one finds the emittances $E=-c_{\mu}$ and $E=c_{\mu}$ for the bar and the disc, respectively. Here, $c_{\mu}$ denotes the relative electro-chemical capacitance between the edge channels. While the emittance of a Corbino disc is an electro-chemical capacitance, the emittance of a quantum Hall bar turns out to be a negative electro-chemical capacitance. The interchange of the sign can be understood intuitively by remarking that the kinetic part $\delta Q^{(k)}$ and the part $\delta Q^{(s)}$ associated with screening are interchanged for the two different topologies. Indeed, for a voltage oscillation at contact 1 of the bar, transmitted charge goes to reservoir 2 and induced charge comes back via edge channel 2. In the Corbino geometry, on the other hand, transmitted charge comes back to contact 1 and screening charge goes to reservoir 2 . 
In order to obtain an intuition for the signs of emittances, consider Fig. 4.a where a two-terminal quantum Hall bar with two pairs of edge channels is shown. A constriction is assumed to bend back the second pair (3 and 4) which will thus not contribute to the dcconductance. The dc-conductance is $e^{2} / h$ as for the case of the bar in Fig. 1.a. However, the second pair gives a capacitive contribution to a time dependent current. From Eq. (21) and using Eq. (8), one finds immediately $E=c_{\mu, 12}-c_{\mu, 34}$. Hence, the emittance is a capacitance (i.e. $E>0$ ) if the Coulomb interaction between edge channels 3 and 4 is stronger than the Coulomb interaction between edge channels 1 and 2. The transmitting edge channels contribute thus with a negative capacitance. It is very remarkable that only two elements of the full capacitance matrix $c_{\mu, k l}$ determine the emittance. This is a consequence of the quantized contact transmission-probabilities and of the symmetry and current-conservation properties of the capacitance matrix. The direct way in which our approach permits to derive this result demonstrates its usefulness.

\section{B. Three-terminal device: a bar with additional gate}

The three-terminal device in Fig. ๑.b consists of a quantum Hall bar with a gate on top of the 2DES and close to one sample edge. The gate is connected to a further contact and couples only capacitively to the edge channels. This setup has been investigated in Ref. [23]. Clearly, all the $G_{\alpha 3}$ and $G_{3 \beta}$ vanish. The dc-conductance for the contacts 1 and 2 is

equal to $G_{\alpha \beta}^{(0)}$ for the quantum Hall bar in Fig. 1.a. The presence of the gate breaks the symmetry of the quantum Hall bar where at equal time the magnetic field $B$ is inverted and the reservoirs 1 and 2 are interchanged. One expects thus that the emittance matrix $\mathbf{E}$ is an asymmetric function of the magnetic field. Equation (21) yields

$$
\mathbf{E}(B)=\left(\begin{array}{ccc}
c_{\mu, 21} & c_{\mu, 22} & c_{\mu, 23} \\
c_{\mu, 11} & c_{\mu, 12} & c_{\mu, 13} \\
c_{\mu, 31} & c_{\mu, 32} & c_{\mu, 33}
\end{array}\right)
$$


For instance, by measuring the current at contact 1 for a voltage oscillation at the gate, one finds $E_{13}(B)=c_{\mu, 23}$ for one polarity of the field $B$, but $E_{13}(-B)=c_{\mu, 31}$ for the other field polarity. This follows directly from the reciprocity relations (23). Because the capacitance $c_{\mu, 13}$ between channels 1 and 3 is different from the capacitance $c_{\mu, 23}$ between channels 2 and 3, one observes a completely asymmetric emittance coefficient $E_{13}(B)$ as a function of the magnetic field. This prediction is in agreement with the experimental results reported in Ref. [23]. The symmetry of the emittance matrix strongly reflects the geometry of the edge-channel arrangement.

\section{Four-terminal Hall bars}

In Fig. 5, four-terminal samples are shown which are used in order to investigate the quantum Hall effect [1]. In such devices, two contacts serve as current source and sink, whereas the two remaining contacts are used as voltage probes. In Fig. 5.a an ideal bar is shown where edge channels connect subsequent contacts along the sample edge. In the sample of Fig. 5.b, on the other hand, there are certain edge channels leaving one and the same contact but connecting different contacts.

Let us assume for the ideal four-terminal bar in Fig. 5.a a filling factor between the integers $p$ and $p+1$ such that $p$ edge channels exist along each sample edge which connect contact $k$ with contact $k+1$. It is possible to define electro-chemical capacitances $c_{\mu, j k}$ between these sets of edge channels which leave contact $k$ and of those which leave contact

$j$. For each of those sets we plotted a single directed line. The Eqs. (14) and (21) yield $G_{\alpha \beta}^{(0)}=$ $g\left(\delta_{\alpha \beta}-\delta_{\alpha-1 \beta}\right)$ for the dc-conductance, and $E_{\alpha \beta}=c_{\mu, \alpha-1 \beta}$ for the emittance, respectively. Here, we defined $g=p\left(e^{2} / h\right)$, and the indices 0 and 4 have to be identified with each other.

On the other hand, for the specific non-ideal Hall bar plotted in Fig. 5.b the connection between contacts via edge channels is not simply determined by the topology of the sample boundary. In the particular case of Fig. 5.b, the dc-conductance becomes 


$$
\mathbf{G}^{(0)}=\frac{e^{2}}{h}\left(\begin{array}{cccc}
2 & 0 & -1 & -1 \\
-1 & 1 & 0 & 0 \\
-1 & -1 & 2 & 0 \\
0 & 0 & -1 & 1
\end{array}\right)
$$

For the emittance coefficients one finds expressions of the form $E_{11}=c_{\mu, 14}+c_{\mu, 17}+c_{\mu, 54}+c_{\mu, 57}$ etc., where the $c_{\mu, k l}$ denote the electro-chemical capacitances between edge channels labelled as shown in Fig. 5.b. Below, we will use this example in order to discuss the effect of voltage probes. Furthermore, we will derive the frequency dependent longitudinal and Hall resistances for the ideal Hall sample in Fig. 5.a.

\section{EFFECT OF VOLTAGE PROBES}

In this section we study the crossover from a $M$-terminal sample to a $M$ - 1-terminal sample by using one contact, say contact $\Omega$, as a voltage probe. For the dc-conductance, this problem has been investigated in Ref. [30. We assume that there is at least one edge channel which connects contact $\Omega$ with a different contact. For an ideal voltage probe, there is no possibility for charge to pass through contact $\Omega$ such that $\delta I_{\Omega} \equiv 0$. By eliminating $\delta V_{\Omega}$ in Eq. (1), one obtains from

$$
\delta V_{\Omega}=-\frac{1}{G_{\Omega \Omega}^{(0)}} \sum_{\beta \neq \Omega}\left(G_{\Omega \beta}^{(0)}-i \omega\left(E_{\Omega \beta}-\frac{G_{\Omega \beta}^{(0)}}{G_{\Omega \Omega}^{(0)}} E_{\Omega \Omega}\right)\right) \delta V_{\beta}
$$

a new admittance $\tilde{G}_{\alpha \beta}(\omega)=\tilde{G}_{\alpha \beta}^{(0)}-i \omega \tilde{E}_{\alpha \beta}$ for the remaining $M-1$ contacts, where

$$
\begin{aligned}
& \tilde{G}_{\alpha \beta}^{(0)}=G_{\alpha \beta}^{(0)}-\frac{G_{\alpha \Omega}^{(0)} G_{\Omega \beta}^{(0)}}{G_{\Omega \Omega}^{(0)}} \\
& \tilde{E}_{\alpha \beta}=E_{\alpha \beta}+\frac{G_{\alpha \Omega}^{(0)} G_{\Omega \beta}^{(0)}}{\left(G_{\Omega \Omega}^{(0)}\right)^{2}} E_{\Omega \Omega}-\frac{G_{\alpha \Omega}^{(0)}}{G_{\Omega \Omega}^{(0)}} E_{\Omega \beta}-E_{\alpha \Omega} \frac{G_{\Omega \beta}^{(0)}}{G_{\Omega \Omega}^{(0)}} .
\end{aligned}
$$

A brief calculation confirms that Eqs. (22) and (23) remain valid for Eqs. (27) and (28). The additional terms appearing in Eqs. (27) and (28) describe scattering between edge 
channels at contact $\Omega$ (incoherent terms) [30]. Now, the probability of a carrier to go from contact $\beta$ to contact $\alpha$ is no longer restricted to the values zero and unity. The additional terms have the following simple interpretations. Firstly, the second term on the right hand side of Eq. (27) describes the equipartition of the current which comes from contact $\beta$ to $\Omega$ between the channels which go from contact $\Omega$ to $\alpha$. Secondly, the three correction terms on the right hand side of Eq. (28) can be associated with processes

1) where carriers go from contact $\beta$ to contact $\alpha$ bypassing $\Omega$ and obtain a 'self-emittance' contribution $E_{\Omega \Omega}$,

2) where carriers induced via the emittance $E_{\Omega \beta}$ are transmitted from contact $\Omega$ to contact $\alpha$, and

3) where carriers which are transmitted from contact $\beta$ to contact $\Omega$ interact via the emittance $E_{\alpha \Omega}$ with contact $\alpha$.

As an example, we consider the four-terminal sample of Fig. 5.b where contact 3 is to serve as the voltage probe. The Eqs. (27) and (28) yield the following three-terminal dc-conductance for the contacts 1,2 , and 4 :

$$
\tilde{\mathbf{G}}^{(0)}=\frac{e^{2}}{h}\left(\begin{array}{ccc}
3 / 2 & -1 / 2 & -1 \\
-1 & 1 & 0 \\
-1 / 2 & -1 / 2 & 1
\end{array}\right) \text {. }
$$

For example, carriers from edge channel 2 will be scattered at contact 3 with probability one half to channel 3 and one half to channel 7 , which implies $\tilde{G}_{42}^{(0)}=\tilde{G}_{12}^{(0)}=-e^{2} / 2 h$. Similar interpretations can be found for the other elements of the dc-conductance matrix (29).

From Eq. (28) one obtains the emittance matrix

$$
\tilde{\mathbf{E}}=\hat{\mathbf{E}}+\left(\begin{array}{ccc}
E_{33} / 4+E_{13} / 2+E_{31} / 2 & E_{33} / 4+E_{13} / 2+E_{32} / 2 & E_{34} / 2 \\
E_{23} / 2 & E_{23} / 2 & 0 \\
E_{33} / 4+E_{43} / 2+E_{31} / 2 & E_{33} / 4+E_{43} / 2+E_{32} / 2 & E_{34} / 2
\end{array}\right) .
$$

where the $3 \times 3$-matrix $\hat{\mathbf{E}}$ is obtained from the matrix $\mathbf{E}$ by deleting row 3 and column 3 . 
The fact that $\tilde{E}_{24}=E_{24}$ holds can be easily understood from Eq. (28): there are neither edge channels which go from contact 4 to 3 nor from contact 3 to 2. Simple interpretations exist also for the other emittance coefficients. For example, consider the additional term $E_{23} / 2$ of $\tilde{E}_{22}$. A voltage oscillation in contact 2 induces a current in edge channel 2 which leads to contact 3. This current is divided into two parts (channels 3 and 7) at contact 3 . Hence, a contribution $E_{23}$ with a factor one half occurs.

\section{LONGITUDINAL AND HALL RESISTANCES AT LOW FREQUENCIES}

The integer quantum Hall effect corresponds to the quantization of the Hall resistance and the vanishing of the longitudinal resistance of the ideal four-probe quantum Hall bar of Fig. 5.a at zero frequency [1]. Two of the contacts serve as voltage probes, whereas the two remaining contacts are used as source and sink for the current. The discussion of the quantum Hall effect in terms of edge channels is provided by Ref. [2]. With the help of the theory presented in this paper, the results of these references can now be extended to the low frequency case.

If the contacts 3 and 4 in Fig. 5.a are the voltage probes, the longitudinal resistance is defined by $R_{L}=R_{12,34}=\left(\delta V_{3}-\delta V_{4}\right) / \delta I_{1}$. On the other hand, the Hall resistance is defined by $R_{H}=R_{13,24}=\left(\delta V_{2}-\delta V_{4}\right) / \delta I_{1}$, provided the contacts 2 and 4 are voltage probes. With the help of Eq. (囵), $R_{L}$ and $R_{H}$ can be expressed in terms of the $G_{\alpha \beta}$. After some linear algebra one finds [2] $R_{L}=\left(G_{32} G_{41}-G_{31} G_{42}\right) / D$ and $R_{H}=\left(G_{21} G_{43}-G_{41} G_{23}\right) / D$, where $D$ is the determinant of the $3 \times 3$ matrix $G_{\alpha \beta}$ restricted to, say, $\alpha, \beta=1, \ldots, 3$. By using the results of Sect. V.c, one obtains a longitudinal resistance $R_{L}=i \omega E_{41} / g^{2}$, where $g=p e^{2} / h$ with $p$ being the number of edge channels along an edge. With $E_{41}=c_{\mu, 13}$ one obtains

$$
R_{L}=i \omega \frac{c_{\mu, 13}}{g^{2}}
$$

The leading term of the longitudinal resistance is determined by the Coulomb coupling between the current circuit and the voltage circuit which are represented by edge channels 1 and 3, respectively. On the other hand, the Hall resistance turns out to be 


$$
R_{H}=\frac{1}{g}+i \omega \frac{c_{\mu, 24}-c_{\mu, 13}}{g^{2}}
$$

This result implies that, in contrast to the longitudinal resistance, for the Hall resistance the sign of the first-order term with respect to frequency depends on the specific locations

of the contacts. In principle, the capacitances $c_{\mu, 24}$ and $c_{\mu, 13}$ can be found independently by measuring $R_{L}$ for appropriate choices of current and voltage probes. A further measurement of $R_{H}$ provides then a test for the validity of Eqs. (31) and (32). Finally, a direct calculation shows that the Eqs. (31) and (32) satisfy the reciprocity relations [2] $R_{j k, m n}(-B)=R_{m n, j k}(B)$; in particular, $R_{L}(-B)=R_{L}(B)$ holds.

\section{SUMMARY}

We investigate the low frequency admittance of quantized Hall samples by using a simple discrete potential model based on the decomposition of the 2DES in (well-separated) metallic and dielectric parts and by applying a general theory of the low frequency admittance $G_{\alpha \beta}=G_{\alpha \beta}^{(0)}-i \omega E_{\alpha \beta}$ for mesoscopic conductors. The main result is an expression for the emittance matrix $E_{\alpha \beta}$ in terms of electro-chemical capacitance elements which depend on the geometrical configuration and the density of states of the edge channels. We emphasize that the theory satisfies the important requirement of charge neutrality and current conservation. The emittance gives the charge emitted through contact $\alpha$ mediated by the Coulomb interaction of edge channels for a voltage-variation at contact $\beta$. If there is no transmission of charge between different reservoirs, the emittance is a capacitance, but in the presence of transmission the emittance can even be a negative capacitance. This has been exemplified by comparing Corbino and bar geometries. The symmetry of the emittance matrix with respect to the magnetic field depends significantly on the geometry of the edge channels. The presence of a voltage probe and the resulting inter edge-channel scattering at the voltage probe is investigated. We finally derive expressions for the frequency dependent longitudinal and Hall resistances of an ideal four-probe bar. Due to the intuitive expression 
for the emittance, all results have simple interpretations.

Acknowledgement This work was supported by the Swiss National Science Foundation.

\section{APPENDIX}

In order to derive the electro-chemical capacitance matrix $c_{\mu, k l}$ for a system with many edge channels, we assume that the spatial variation of the electrostatic potential inside the edge channels can be neglected (discrete potential model). Then, Eq. (丑) remains valid and the definition of a geometrical capacitance $c_{j k}$ is meaningful. It is convenient to use vector and matrix notation. Let us write the $N \times N$-matrix for the geometrical capacitance of the (disconnected) edge channels by $\mathbf{c} \equiv c_{j k}$, and in a similar way for the electro-chemical capacitance $\mathbf{c}_{\mu} \equiv c_{\mu, j k}$, and the DOS $\mathbf{D} \equiv D_{k} \delta_{j k}$. We introduce $N$-dimensional vectors $\delta \mathbf{q}$, $\delta \mathbf{U}$, and $\delta \mathbf{V}$ for the charges, the electrostatic and the electro-chemical potentials of the edge channels, respectively. The solution of the Poisson equation for a given charge distribution yields an electric potential $\delta \mathbf{U}=\mathbf{c}^{-1} \delta \mathbf{q}+\delta U^{(0)} \mathbf{1}$, where $\mathbf{1}$ is a vector with all components being unity. Note that a constant potential shift $\delta U^{(0)}$ in the whole sample is always a solution of the Poisson equation and is determined by charge conservation, $\sum_{k} \delta q_{k}=0$. Hence, $\delta U^{(0)}=\sum_{j, k} c_{j k} \delta U_{k} / \sum_{j, k} c_{j k}$ which defines a matrix $\boldsymbol{\Lambda}$ such that $\delta U^{(0)} \mathbf{1}=\boldsymbol{\Lambda} \delta \mathbf{U}$. The electro-chemical capacitance matrix $\delta \mathbf{q} / \delta \mathbf{V}$ follows from $\delta \mathbf{q}=\mathbf{D}(\delta \mathbf{V}-\delta \mathbf{U})=\mathbf{c}(\delta \mathbf{U}-$ $\left.\delta U^{(0)} \mathbf{1}\right)$ and can be expressed in the form

$$
\mathbf{c}_{\mu}=\left(\mathbf{c}^{-1}+(\mathbf{I}-\mathbf{\Lambda}) \mathbf{D}^{-1}\right)^{-1}(\mathbf{I}-\mathbf{\Lambda})
$$

where I denotes the identity matrix. 


\section{REFERENCES}

[1] K. von Klitzing, G. Dorda, and M. Pepper, Phys. Rev. Lett. 45, 494 (1980).

[2] M. Büttiker, Phys. Rev. B 38, 9375 (1988).

[3] For reviews see R. J. Haug, Semicond. Sci. Technol. 8, 131, (1993); and Semiconductors and Semimetals, Vol. 35, edited by M. Reed, Academic Press, Boston, (1992).

[4] M. Büttiker, H. Thomas, and A. Pretre, Phys. Lett. A 180, 364 (1993); Z. Phys. B 94, 133 (1994).

[5] M. Büttiker, J. Phys.: Condensed Matter 5, 9361 (1993).

[6] B. I. Halperin, Phys. Rev. B 25, 2185 (1982).

[7] D. B. Chklovskii, B. I. Shklovskii, and L. I. Glazman, Phys. Rev. B 46, 4026 (1992).

[8] N. R. Cooper and J. T. Chalker Phys. Rev. B 48, 4530 (1993).

[9] K. Lier and R. R. Gerhardts, Phys. Rev. B 50, 7757 (1994).

[10] A. L. Efros, Solid State Commun. 65, 1019 (1988); Solid State Commun. 70, 253 (1989). Solid State Commun. 70, 253 (1989)

[11] A. M. Chang, Solid State Commun. 74, 871 (1990).

[12] C. W. J. Beenakker, Phys. Rev. Lett. 64, 216 (1990).

[13] B. Y. Gelfand and B. I. Halperin, Phys. Rev. B 49, 1862 (1994).

[14] C. Méndez and J. Simonin, Phys. Rev. B 49, 17199 (1994).

[15] B. J. van Wees Phys. Rev. Lett. 66, 2033 (1991).

[16] B. Jeanneret et al., Phys. Rev. B 51, 9752 (1995).

[17] A. H. McDonald, T. M. Rice, and W. F. Brinkman, Phys. Rev. B 28, 3648 (1983).

[18] D. B. Chklovskii, Phys. Rev. B 51, 9895 (1995). 
[19] For the fractional quantized Hall effect a widely discussed alternative description of edge states is based on chiral Luttinger liquids (see X. G. Wen, Phys. Rev. B43, 11025 (1991)). This approach neglects electrostatic restructuring but can be used to discuss the role of contacts (see C. L. Kane and M. P. A. Fisher (unpublished)).

[20] A. O. Govorov, Phys. Rev. 51, 14498 (1995).

[21] L. D. Landau and E. M. Lifschitz, (Pergamon Press, Oxford, 1980). Vol. VIII

[22] H. Hirai and S. Komiyama, Phys. Rev. B 49, 14012 (1994).

[23] W. Chen, T. P. Smith III, M. Büttiker, and M. Shayegan, Phys. Rev. Lett. 73, 146 (1994).

[24] S. Komiyama and H. Hirai, (unpublished)

[25] D. J. Thouless, Phys. Rev. Lett. 71, 1879 (1993).

[26] V. B. Shikin, JETP Lett. 61, 227 (1995).

[27] B. J. van Wees et al., Phys. Rev. B43, 12431 (1991).

[28] G. Müller et al., Phys. Rev. B42, 7633 (1990).

[29] A. Pretre, H. Thomas, and M. Büttiker, (unpublished).

[30] M. Büttiker, IBM J. Res. Develop. 32, 317 (1988). 


\section{FIGURES}

FIG. 1. a) Quantum Hall bar with a single pair of edge channels (thin directed lines) and connected to two reservoirs at electro-chemical potentials $\mu_{1,2}$. b) Corbino disc with contacts at the inner and the outer edges.

FIG. 2. a) Single particle potential for a transverse cross section of the Hall bar in Fig. 1.a. Empty, partially filled, and filled circles correspond to empty, partially filled, and filled states, respectively. Edge channels are the partially filled and extended states at the Fermi energy $E_{F}$ close to the sample edge, where the potential is dominated by the confinement potential. b) Nonequilibrium version of part a. The electro-chemical voltage variation $\delta V_{1}$ induces charges $\delta q_{1,2}$ and nonequilibrium electrostatic potential shifts $\delta U_{1,2}$ in the edge channels.

FIG. 3. An emittance element $E_{\alpha \beta}$ is the sum over all electro-chemical capacitance elements $c_{\mu, k l}$ of the edge channels $k$ and $l$ which correspond to the elementary process shown in this figure.

FIG. 4. a) Two-terminal bar with constriction. Only one pair of edge channels connects different reservoirs, whereas another pair returns to the original reservoir. b) Three-terminal Hall bar with gate (3). The asymmetric geometry leads to an asymmetric magneto-capacitance as a function of the magnetic field.

FIG. 5. a) Ideal four-terminal Hall bar. The geometry of edge channels is determined by the sample boundary. b) Four-terminal Hall bar with complicated edge-channel arrangement. 




b)

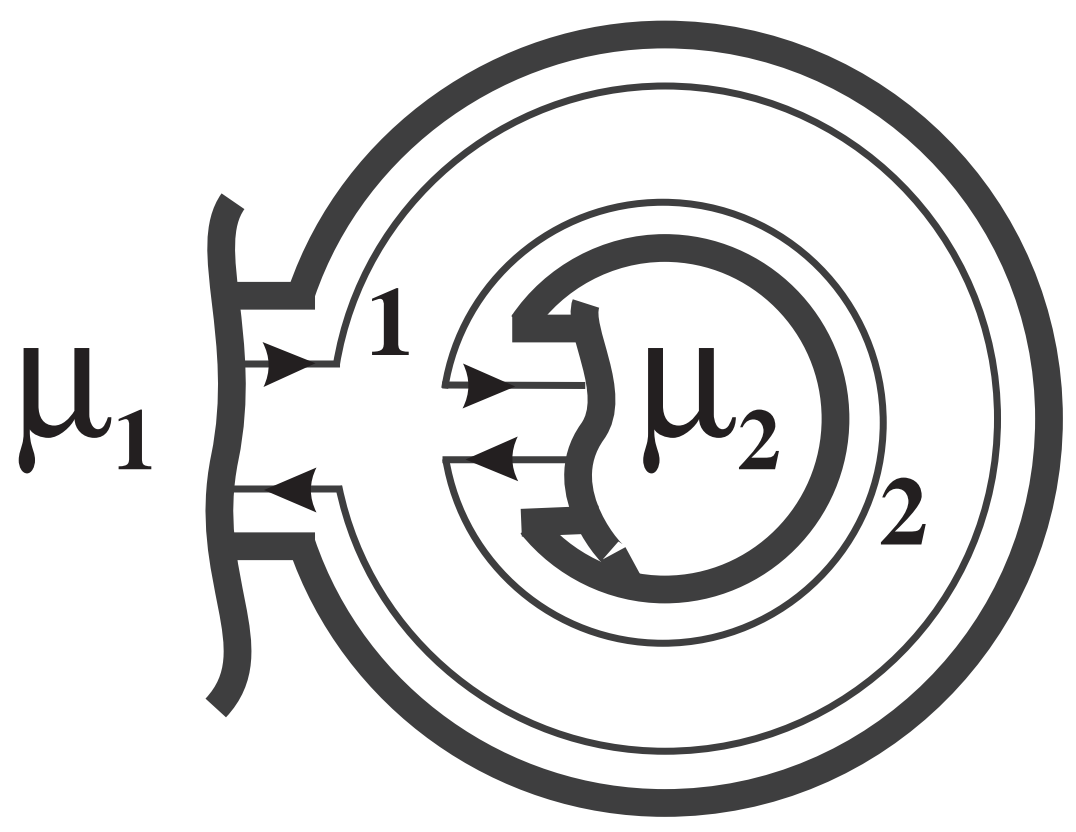



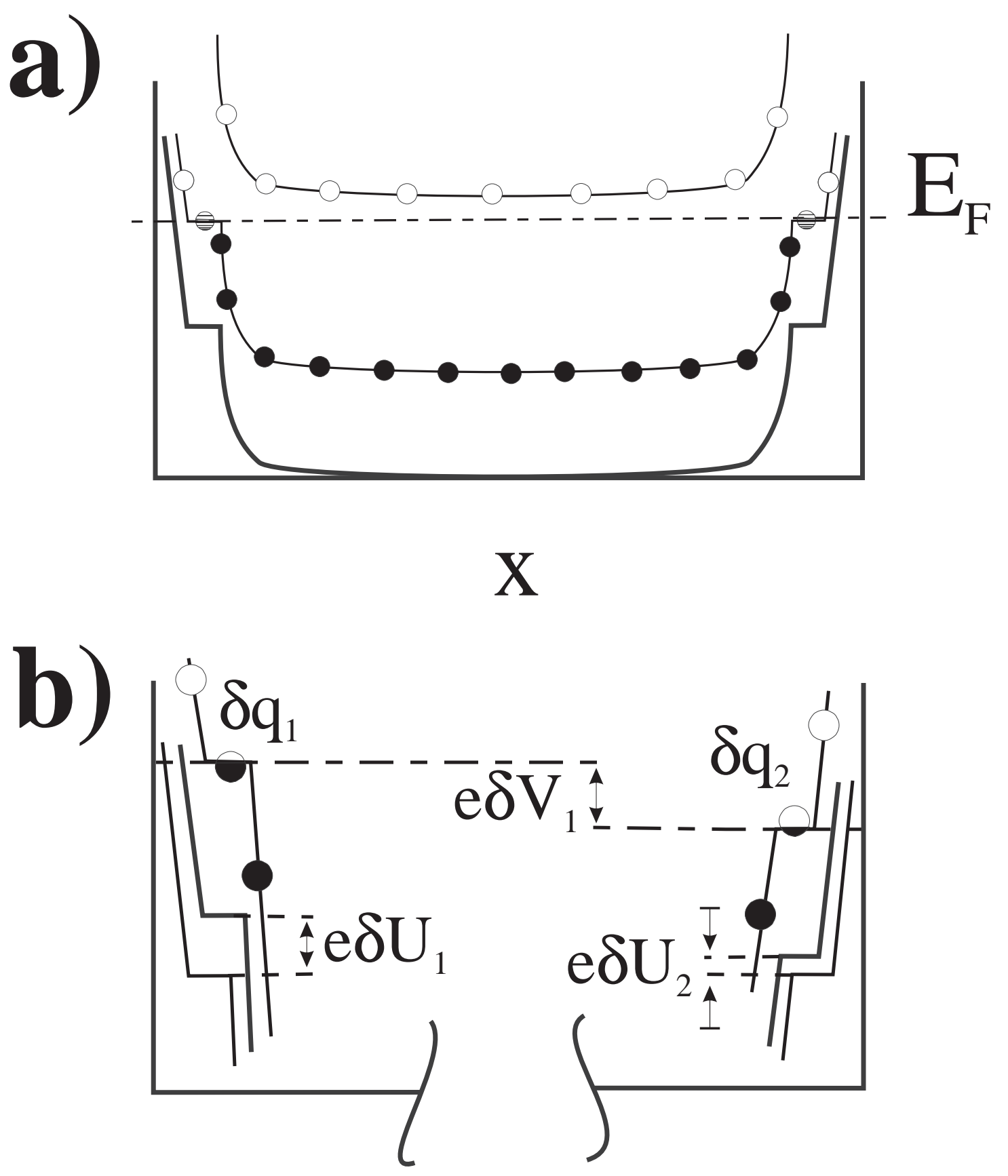


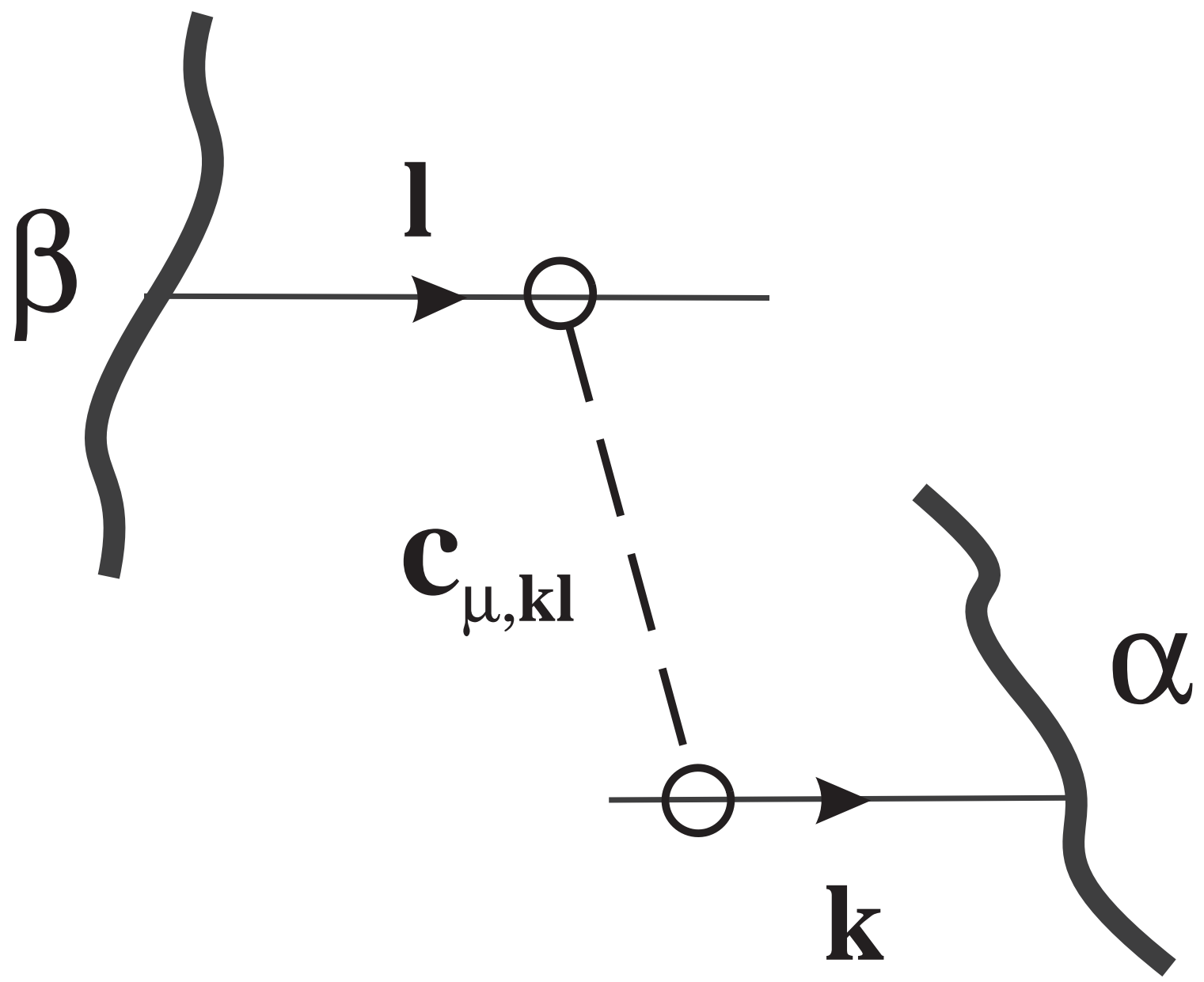




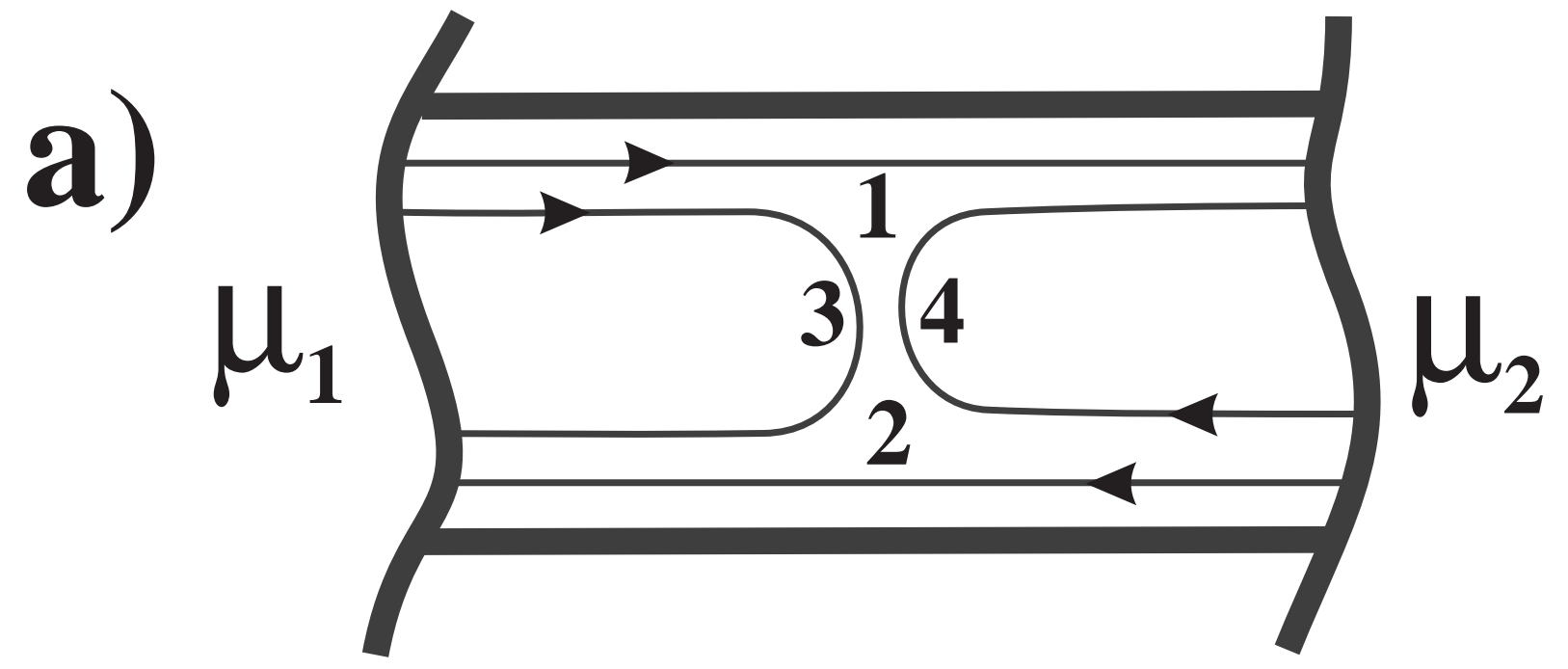

b)

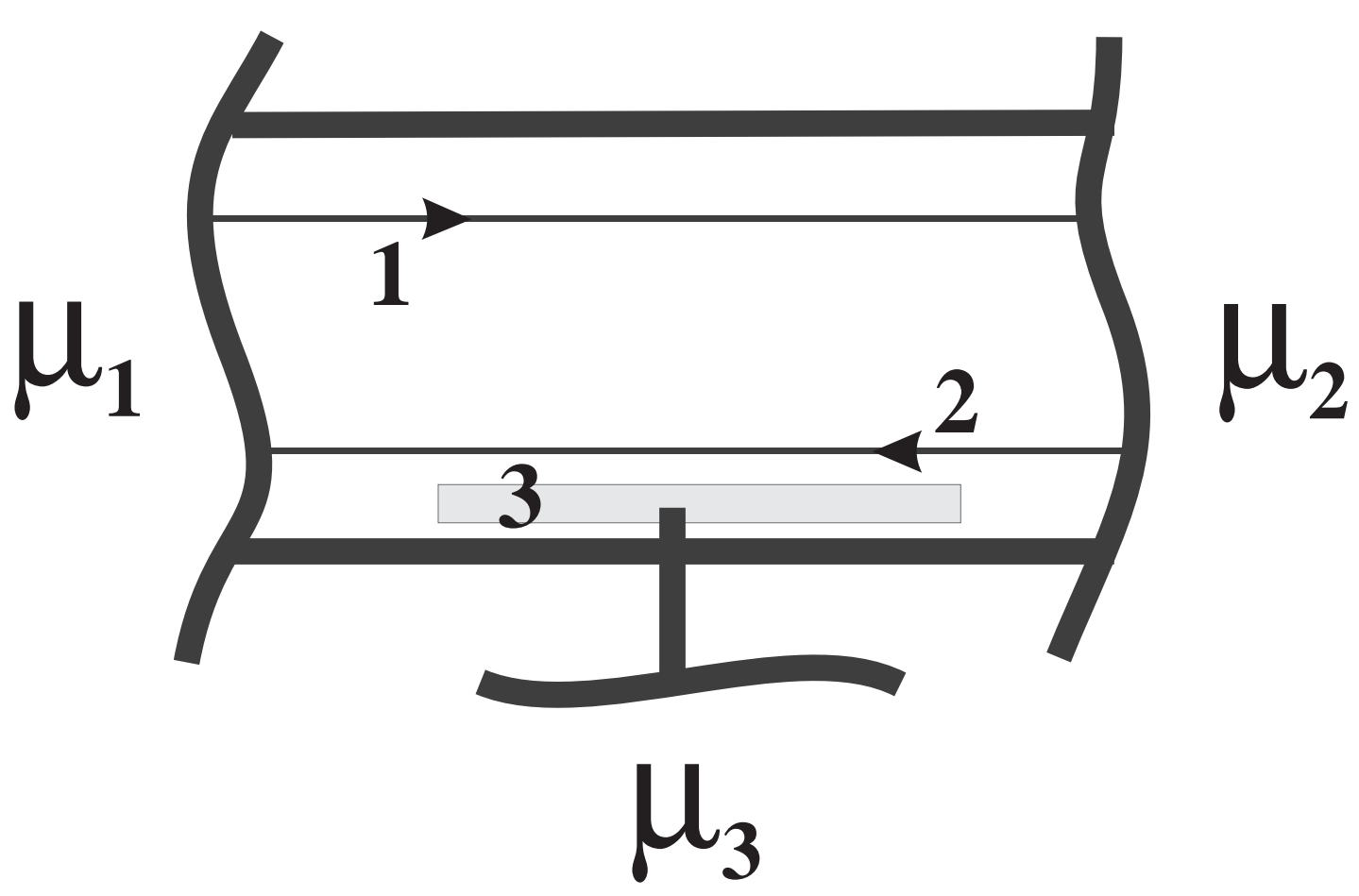


a)

$\mu_{2}$



b)

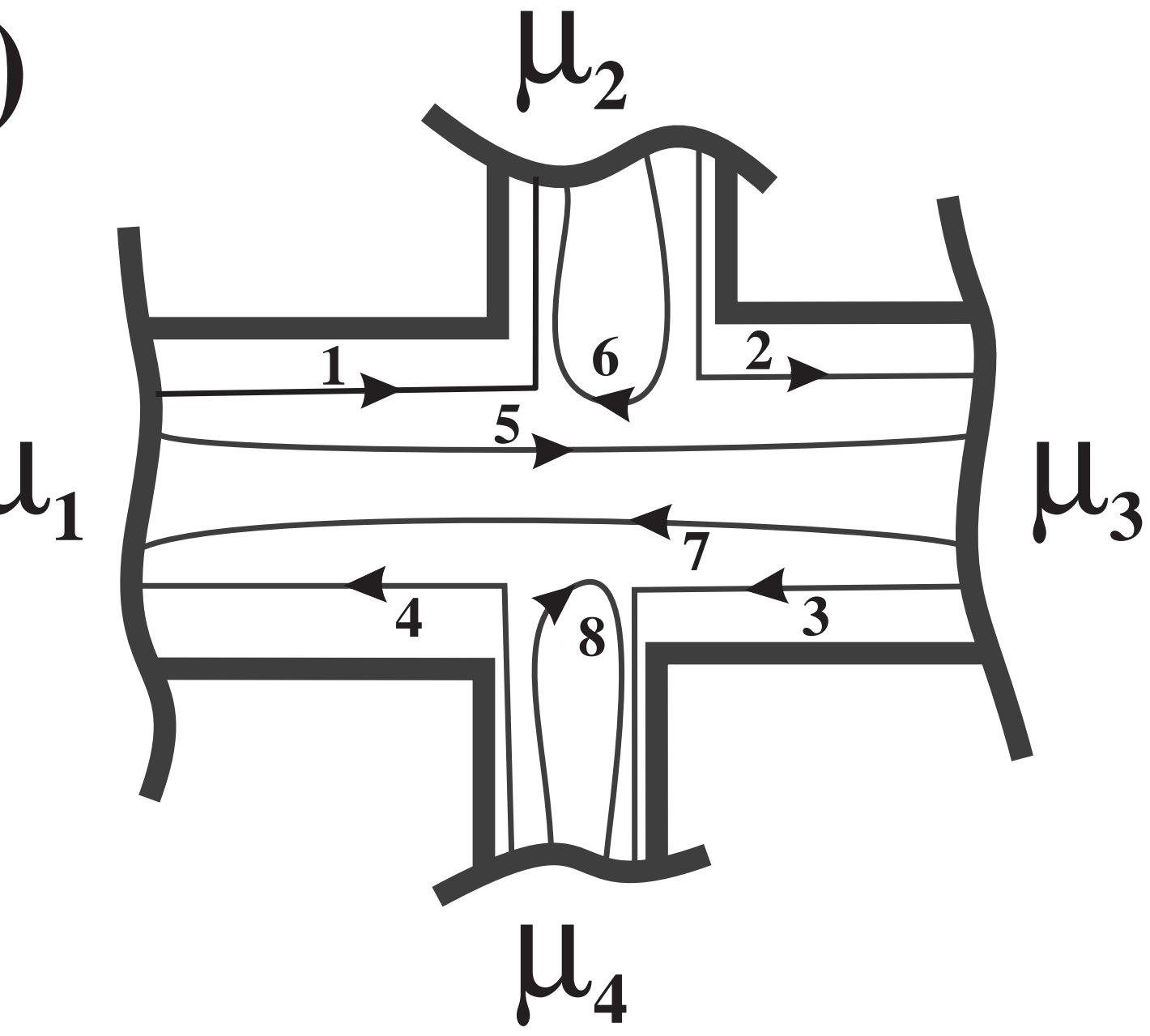

